\title{
Dental anomalies - what is referred? And what should be?
}

\section{The orthodontic-oral surgery interface. Parts 1 and 2}

Part 1: Br Dent J 2018; 225: 431-435; http://dx.doi.org/10.1038/sj.bdj.2018.739. Part 2: Br Dent J 2018; 225: 491-496; http://dx.doi.org/10.1038/sj.bdj.2018.751

Patients with complex dental anomalies can be challenging to treat. Often, with their unique dental needs, comes extensive treatment planning and multidisciplinary care. Hence, general dental practitioners (GDPs) need to have the knowledge and skill base to accurately identify and refer such patients. However, what dental anomalies are commonly referred? And how should they be managed?

To explore these questions, Owaise Sharif and co-authors carried out a service evaluation of the joint dento-alveolar (JDA) clinic at the Croydon University Hospital. A prospective patient note analysis was carried out of 100 new patients attending between November 2014 and September 2015. Fifty-four percent of the patients were female, with the mean age 17 years. The most common anomalies referred were:

- Impacted canines (49\%)

- Supernumeraries (16\%)

- Impacted second premolars (10\%).

Others included low lying frenulum, caries, cysts, tongue tie etc. It was found that orthodontic specialists provided $79 \%$ of the referrals followed by GDPs at $20 \%$ and the district hospital at $1 \%$. Out of these 100 patients, 92 of them were internally referred from the JDA clinic to the orthodontic department, leaving only eight patients being referred to the oral surgery department.

In addition to the service analysis described in part 1 of the $B D J$ series, in part 2 the authors
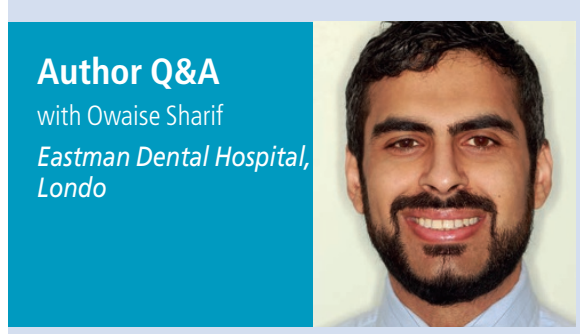

Why do GDPs need to know about complex dental anomalies?

Dental anomalies are often asymptomatic and may develop from an early age, the GDP therefore has a crucial role in early identification and management. In addition, dental

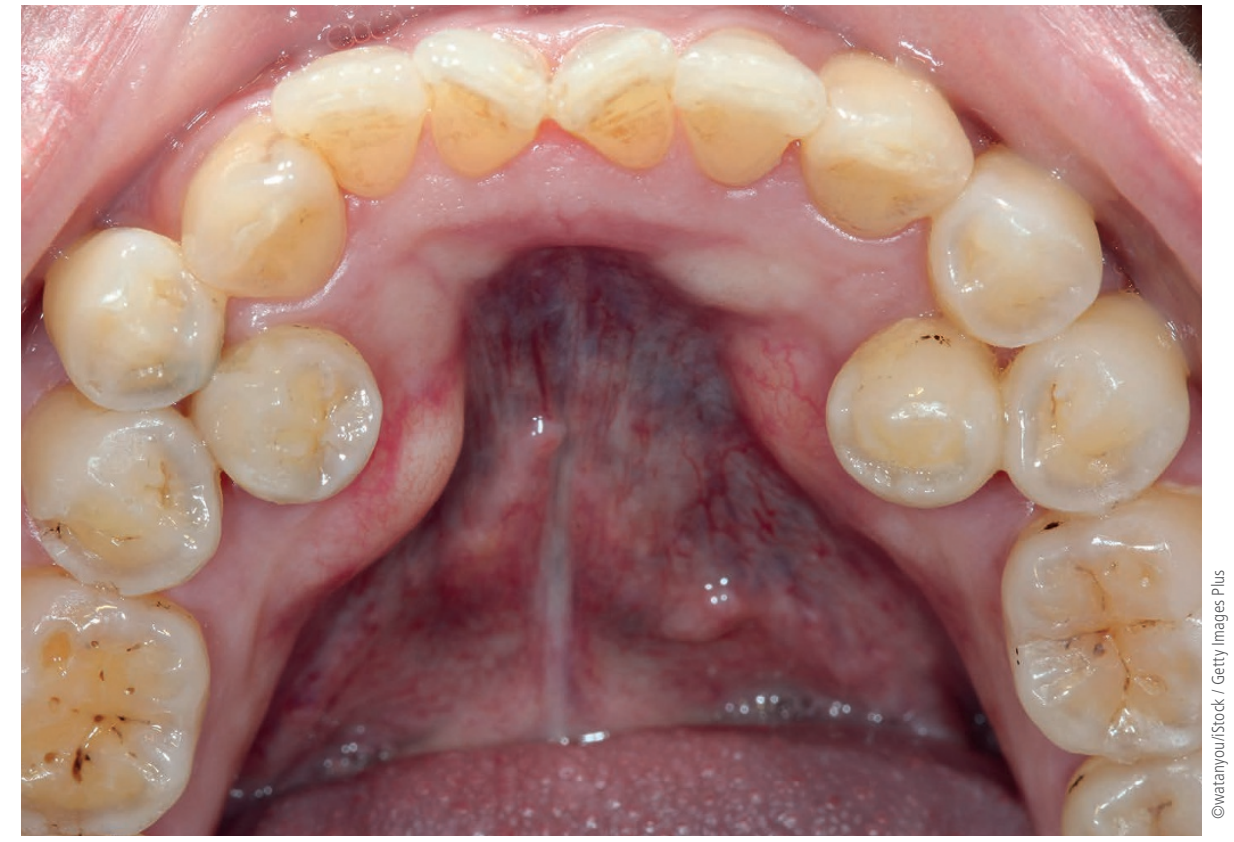

provide an overview of common dental anomalies. Their definition, classification, aetiology and prevalence have been outlined along with their diagnosis and management. The aim of this is to increase awareness amongst practitioners and help them formulate appropriate treatment plans. Part 1 of the series covers soft tissue anomalies, dentigerous cysts, transpositions and supernumerary teeth. Whereas part 2 focuses on impacted teeth, delayed and failed eruption, ankylosed incisors and infra-occluded deciduous molars.

anomalies often require onward referral to a specialist for management and so knowledge of potential management strategies will aid the GDP in counselling patients.

\section{Why was it important to run this service} evaluation?

The number of referrals to this service has been increasing in recent years and previous audits have resulted in additional clinical sessions being allocated to allow the service to run more efficiently. This service evaluation provided an overview of the presenting anomalies and highlighted the continued appropriateness of referrals to the service.
In conclusion, the articles highlight the importance of the general dental practitioner's role in monitoring patients' dental development and identifying early signs of common variances. It is crucial they refer patients to the appropriate specialist service at the right time, be it for an opinion or for treatment. In the same breath, the role of orthodontic-oral surgery department is equally as important when it comes to providing care to such patients.

By Sidra Suleman

Does the system need improvement to better diagnose and treat anomalies? The average age of patients presenting to the clinic with impacted canines was 17 years, this is a late presentation age. Impacted canines are a common dental anomaly and although there are number of reasons for late referral, this service evaluation has highlighted an area where knowledge improvement may be beneficial. The age of presentation for unerupted central incisors was also late. We hope that the information presented in this two part series is useful for the whole dental team. 